\title{
Awake postural change to prone in a morbidly obese patient
}

\author{
Tetsuro Kimura · Takayuki Katsuragawa • \\ Shigehito Sato
}

Received: 15 April 2014 / Accepted: 29 October 2014 / Published online: 24 December 2014

(C) Japanese Society of Anesthesiologists 2014

\section{To the Editor:}

In prone position surgery for obese patients, body position change after induction of general anesthesia is very stressful for staff in the operating room [1]. There are risks of a fall from the operating table during body position change, and of interruption of blood circulation and peripheral nerve injury due to an improper position.

We report a case of successful anesthetic management in spine surgery performed in a prone position for an obese patient with a body mass index of $65 \mathrm{~kg} / \mathrm{m}^{2}$. Posterior decompression was scheduled for a diagnosis of thoraciclumbar ossification of the posterior longitudinal ligament. To simulate the preoperative situation, we asked the patient to adopt a prone position and adjust to an optimal position by himself on the day before the surgery.

As a difficult airway was anticipated, the trachea was intubated in a conscious state. After awake tracheal intubation, the patient was able to adopt the prone position, adjust to the optimal position by himself, and verify the site of least compression. This made it possible to minimize pressure damage in the contact portion of the skin and operating table, reduce compression of nerves and vessels, and decrease the effect of thoracic compression on respiration. General anesthesia was then achieved using total intravenous anesthesia with propofol and remifentanil, with maintenance of the bispectral index at 40-60 through adjustment of the propofol dose. Waking from anesthesia was rapid and smooth after an operation time of $5 \mathrm{~h}$. There was no intraoperative waking or pressure findings.

We performed postural change smoothly by a preoperative simulation. Awake postural change in an intubated state before prone position surgery in morbidly obese patients is considered as one of the options.

\section{Reference}

1. Brodsky JB, Oldroyd M, Winfield HN, Kozlowski PM. Morbid obesity and the prone position: a case report. J Clin Anesth. 2001;13:138-40.
T. Kimura $(\bowtie) \cdot$ T. Katsuragawa $\cdot$ S. Sato

Department of Anesthesiology and Intensive Care, Hamamatsu

University School of Medicine, 1-20-1 Handayama, Higashi-ku,

Hamamatsu, Shizuoka 431-3192, Japan

e-mail: kimutetsuuu@hotmail.com

T. Katsuragawa

e-mail: tkykktr@yahoo.co.jp

S. Sato

e-mail: shige-15@hama-med.ac.jp 